\title{
ACESSO ABERTO À INFORMAÇÃO CIENTÍFICA: POLÍTICAS E INICIATIVAS GOVERNAMENTAIS
}

\section{ACCESO ABIERTO A LA INFORMACIÓN CIENTÍFICA: POLÍTICAS E INICIATIVAS GOBERNAMENTALES}

Terezinha Elisabeth da Silva - telis@uel.br Doutora em Multimeios pela Unicamp. Professora Adjunto da Universidade Estadual de Londrina

\begin{abstract}
Adriana Rosecler Alcará - alcara@uel.br Doutoranda em Avaliação em Psicologia Educacional pela Universidade São Francisco. Mestre em Educação pela Universidade Estadual de Londrina. Professora Assistente da Universidade Estadual de Londrina
\end{abstract}

\section{Resumo}

Trata das políticas de acesso aberto à informação científica e das propostas de ação com ênfase para as iniciativas governamentais em diferentes países. Descreve as recomendações da Unesco e da IFLA. Identifica as principais iniciativas, ações e recomendações internacionais. Finaliza evidenciando peculiaridades de áreas e de formas de tratamento da questão nas políticas já estabelecidas.

\section{Palavras-chave}

Acesso Aberto. Informação científica. Políticas de Informação.

\section{INTRODUÇÃO}

Desde que na década de 1990 vieram à tona as primeiras iniciativas formais e organizadas - em associações, alianças, coalizões - em torno do acesso aberto, não param de crescer os trabalhos e esforços dessas organizações em prol da "filosofia aberta" (COSTA, 2007). Entretanto, é sabido que, se nas organizações não-governamentais - em especial aquelas que integram pesquisadores - o movimento recebe adesões imediatas, no âmbito governamental o que se percebe é uma demora no estabelecimento de infraestrutura física, administrativa e, principalmente, legal que propicie a efetiva aplicação das recomendações emanadas de vários encontros e eventos em nível mundial. 
Criada em 1999, na Convenção de Santa Fé (EUA), a Open Archives Initiative (OAI), define os princípios básicos do acesso aberto à produção científica. A partir desse marco, importantes declarações de apoio ao acesso aberto promoveram a disseminação dessa filosofia, como por exemplo, as declarações de Budapeste, Bethesda e Berlin.

A Budapeste Open Access Initiative $(\mathrm{BOAl})^{1}$ foi criada em fevereiro de 2002, a partir da reunião promovida pelo Open Society Institute (OSI), da Soros Fundation, com o propósito de analisar como as iniciativas isoladas poderiam trabalhar conjuntamente e como o OSI e as demais fundações poderiam utilizar de forma mais efetiva seus recursos para contribuir com o acesso aberto. A declaração recomenda duas estratégias - auto-arquivamento e o acesso aberto aos periódicos.

Em abril de 2003 ocorre uma reunião no Howard Hughes Medical Institute, da qual origina a Bethesda Statement on Open Access Publishing, ${ }^{2}$ com o objetivo de estimular a discussão do acesso aberto na comunidade de pesquisadores da área biomédica, e ainda de delinear princípios para obter apoio formal das agências de financiamento, sociedades científicas, editores, bibliotecários e pesquisadores para publicação dos resultados das pesquisas científicas.

A Berlin Declaration on Open Access to Knowledge in the Sciences and Humanities, ${ }^{3}$ de outubro de 2003, foi estabelecida por representantes de importantes instituições científicas européias, entre elas a Sociedade Max Planck (Alemanha) ${ }^{4}$ e o Centre National de la Recherche Scientifique (CNRS), da França. Baseada e de acordo com as declarações de Budapeste e Bethesda, a Declaração de Berlin recomenda, dentre outras coisas, o incentivo aos pesquisadores para que publiquem seus trabalhos dentro da filosofia do acesso aberto. Reforça também a necessidade de avaliação da produção disponível em acesso aberto, visando assegurar os padrões de qualidade.

Essas iniciativas fizeram com que o movimento de acesso aberto à informação científica ganhasse proporções mundiais e recebesse apoio de diferentes organizações que têm o objetivo de conscientizar universidades, pesquisadores e demais produtores de informação científica a inserirem-se nessa nova filosofia.

Observa-se que as iniciativas em favor do acesso aberto encontram um ponto nevrálgico e de difícil solução: as políticas de informação. A intenção deste artigo é verificar o andamento das iniciativas políticas em nível internacional a partir de duas fontes cronológicas que listam eventos acerca do movimento de acesso aberto: 1) a Timeline of the Open Access Movement, de Peter Suber; 2) Le libre accès à l'information scientifique et technique, mantido pelo INIST/CNRS. Dessas duas fontes foram destacadas as iniciativas mais significativas, principalmente as governamentais, que foram sistematizadas por países. Cada um desses agrupamentos foi detalhado e

\footnotetext{
${ }^{1} \mathrm{http}: / /$ www.soros.org/openaccess/read.shtml

${ }^{2} \mathrm{http}: / /$ www.earlham.edu/ peters/fos/bethesda.htm

${ }^{3} \mathrm{http}: / /$ oa.mpg.de/openaccess-berlin/berlindeclaration.html

${ }^{4}$ http://www.mpg.de
} 
descrito a partir outras fontes que forneceram mais informações a respeito das iniciativas mencionadas.

É importante destacar a variação terminológica concernente às iniciativas de acesso aberto. Muitas vezes, acesso aberto (open access), arquivos abertos (open archives) e acesso livre (free access) à informação aparecem como sinônimos; tecnicamente não são. Entretanto, como não serão tratadas de questões relativas à interoperabilidade, coleta de metadados, disponibilidade de textos integrais e acesso e uso de informações, ao longo deste texto será utilizada a expressão "acesso aberto" para representar aqueles três aspectos, fundamentais na implementação de sistemas de compartilhamento de informação que aderem à "filosofia aberta".

Os resultados aqui apresentados agregam-se a outras pesquisas do projeto de pesquisa "Gestão da Informação da Produção Intelectual da Universidade Estadual de Londrina", desenvolvido no Departamento de Ciência da Informação dessa Universidade, financiado, em parte, com recursos do CNPq.

\section{UNESCO E IFLA: INCIATIVAS SUPRANACIONAIS}

Desde o final da década de 1990 a UNESCO se integrou à defesa do acesso aberto por intermédio de declarações, programas e financiamentos específicos para a implantação de ações. Em 1999, na Conferência Mundial do International Council of Science (ICSU) de Budapeste, foi signatária da Declaration of Science and the Use of Science Knowledge, ${ }^{5}$ que contem 46 itens de considerações e recomendações em torno da relação entre comunidade científica e sociedade e a importância da informação e do conhecimento científico nessa relação.

A UNESCO e a IFLA tornaram público, em 2006, o IFLA/UNESCO Internet Manifesto Guidelines, como meio de implementação das recomendações do IFLA Internet Manifesto (2002). Em 2007 a UNESCO assina a Declaração de Cronenberg (Kronberg Declaration on the Future of Knowledge Acquisition and Sharing) ${ }^{6}$

Publicado pela UNESCO em abril de 2008, o livro Open access to knowledge and information: scholarly literature and digital library initiatives; the South Asian scenario, ${ }^{7}$ de Anup Kumar Das, mostra o cenário das iniciativas de acesso aberto no sul da Ásia, listando e descrevendo periódicos de acesso aberto, coleta de metadados, repositórios institucionais. Segundo o preâmbulo, o livro "pode ser considerado como fonte oficial para o desenvolvimento de acesso aberto na região" (JOSIAH, 2008), e, por conseguinte, para regiões com características similares e países emergentes de modo geral.

\footnotetext{
${ }^{5} \mathrm{http} / / /$ www.unesco.org/science/wcs/eng/declaration_e.htm

${ }_{7}^{6} \mathrm{http}: / /$ portal.unesco.org/ci/en/files/25109/11860402019Kronberg_Declaration.pdf/Kronberg\%2BDeclaration.pdf

${ }^{7}$ http://unesdoc.unesco.org/images/0015/001585/158585e.pdf
} 
Como iniciativa da International Federation of Library Associations and Institutions (IFLA), ressaltamos a Declaração da IFLA sobre o acesso aberto à literatura acadêmica e documentação de pesquisa, publicada em dezembro de 2003. O documento evidencia que o amplo acesso aberto à literatura científica é imprescindível para a compreensão do nosso mundo e para a identificação de soluções aos desafios globais e, particularmente, para a redução da desigualdade de acesso à informação. Alerta que o acesso aberto garante a integridade do sistema de comunicação científica, já que assegura que todos os resultados de pesquisa estarão disponíveis permanentemente para o irrestrito exame e, quando relevantes, elaboração ou refutação (IFLA, 2003).

O documento da IFLA destaca ainda o reconhecimento do papel desempenhado por todos os atores - autores, editores, bibliotecas e instituições - envolvidos no processo de registro e disseminação da pesquisa e define a adoção dos seguintes princípios do acesso aberto: a) o reconhecimento e a defesa dos direitos autorais, em especial dos direitos de atribuição e integridade; b) a adoção do processo de revisão por pares, visando assegurar a qualidade da literatura acadêmica, independente da forma de publicação; c) a oposição à censura governamental, comercial ou institucional das publicações originadas de pesquisas e bolsas de estudos; d) a disponibilização em formato de domínio público de toda a literatura acadêmica e documentos de pesquisa após a expiração do copyright determinado pela lei; e) a implementação de medidas para superar a desigualdade de acesso à informação; f) o apoio às iniciativas que promovem o desenvolvimento de modelos de publicação de acesso aberto sustentáveis; e g) a implementação de mecanismos legais, contratuais e técnicos para assegurar a preservação e a disponibilidade perpétua, usabilidade e autenticidade de toda a literatura acadêmica e documentação de pesquisa (IFLA, 2003).

\section{ESTADOS UNIDOS}

Peter Suber (2006) pontua as dez principais iniciativas relativas ao acesso aberto nos Estados Unidos, destacando, dentre elas, os já históricos ArXiv, The Public Library of Science (PLOS), a liderança do Massachusetts Institute of Technology (MIT) e da Hewlett-Packard no desenvolvimento do DSpace. Fundamentais também na defesa do acesso aberto são algumas organizações cujas ações definiram os rumos das políticas públicas (governamentais) nos Estados Unidos e mesmo em nível mundial. Neste caso, não se pode omitir a atuação da Scholarly Publishing and Academic Resources Coalition (SPARC), da Alliance for Taxpayer Access (ATA), do Open Access Working Group (OAWG) e do Public Knowledge (PK).

Ainda que emanadas de organizações não-governamentais, as derivações dessas iniciativas foram fundamentais para que o governo americano se sensibilizasse para a importância do acesso aberto. Suber (2006) pontua que a lição que os Estados Unidos podem dar aos outros países é que os governos que considerarem a obrigatoriedade do acesso aberto às pesquisas financiadas por verbas públicas enfrentarão muitos lobbies dos editores e precisam se organizar, 
informar e buscar embasamento na defesa do acesso aberto de forma a responder as objeções e assim educar quem elabora as políticas a respeito.

A iniciativa de maior visibilidade nos Estados Unidos foi a definição e o estabelecimento formal de uma política governamental de obrigatoriedade de depósito no repositório de acesso aberto, PubMed Central (PMC), de todo resultado de pesquisa financiada pelo National Institute of Health (NIH). ${ }^{8}$ A lei que determina o procedimento foi assinada pelo presidente George W. Bush em 26 de dezembro de 2007. Tramitou por três anos, desde que, em 2004, o Congresso Americano solicitou que o NIH desenvolvesse uma política de obrigatoriedade de depósito em repositórios de acesso aberto.

Os 561 periódicos listados no site do $\mathrm{NIH}^{9}$ resultam de esforços de representantes de vários segmentos da sociedade americana, como os 25 prêmios Nobel que foram signatários de uma carta aberta ao Congresso Americano em favor do acesso aberto aos resultados de pesquisas financiadas pelo $\mathrm{NIH} .{ }^{10} \mathrm{O} \mathrm{NIH}$ possui hoje os meios para promover o acesso às pesquisas financiadas pelos contribuintes, por intermédio da Biblioteca Nacional de Medicina. A partir da aprovação da proposta pelo Congresso, os financiados pelo NIH ficam obrigados a fornecer à Biblioteca uma cópia eletrônica da versão final de seus trabalhos aceitos para publicação em periódicos científicos, após a revisão pelos pares (AN OPEN..., 2005).

A Association of Research Library (ARL) - que congrega 124 bibliotecas de instituições de pesquisa americanas -, juntamente com outras organizações não-governamentais, engajou-se na busca de abertura de acesso para a informação produzida no âmbito governamental, por intermédio do Federal Depository Library Program e do incentivo à liberdade de informação e ao acesso às pesquisas financiadas pelos fundos públicos (ASSOCIATION OF RESEARCH LIBRARY, 2009).

Criada em 2004 no seio do SPARC e inspirada pelos princípios da BOAl, a Alliance for Taxpayer Access (ATA), agrega 97 instituições representativas de associações científicas, de pesquisadores e cidadãos americanos que se uniram em favor do acesso aberto e irrestrito aos resultados de pesquisas financiadas pelos órgãos governamentais, uma vez que as barreiras criadas pelas assinaturas limitam o acesso do contribuinte americano às pesquisas financiadas com fundos públicos (ALLIANCE FOR TAXPAYER ACCESS, 2009). O acesso do contribuinte (taxpayer access) a essas pesquisas deve ser livre de qualquer custo, porque elas são, de fato, financiadas pelos contribuintes (taxpayer-funded).

\footnotetext{
${ }^{8}$ http://www.nih.gov

${ }^{9}$ http://publicaccess.nih.gov/journal_list.txt

${ }^{10}$ Em maio de 2008, mês do início efetivo da política de obrigatoriedade, já havia 355 periódicos listados no site do $\mathrm{NIH}$.
} 
No âmbito da National Science Foundation (NSF), ${ }^{11}$ o Office of Cyberinfrastructure, vem realizando, desde 2005, vários encontros de seu Comitê Consultivo, com o objetivo de discutir a situação da infraestrutura para pesquisa em ciência e tecnologia nos Estados Unidos, incluindo o acesso à informação e a gestão dos conjuntos de dados digitais. As ações do incluem: a) promoção da interoperabilidade entre conjuntos de dados de várias organizações; b) prevenção de proteção e preservação adequadas em longo prazo, estabelecendo confiabilidade desses dados; c) propiciar bom desempenho, promover confiabilidade e mobilidade de dados por intermédio de ferramentas, tecnologias e serviços compartilhados; e) abrigar preferências específicas geradas pelas comunidades. É também objetivo da NSF desenvolver políticas que enfatizem o acesso aberto e a gestão eficaz desses conjuntos de dados digitais, de acordo com as necessidades das áreas de ciência e tecnologia (NATIONAL SCIENCE FOUNDATION, 2009).

\section{COMISSÃO EUROPEIA}

As ações desenvolvidas pela Comissão Européia em favor da filosofia aberta têm sido tão intensas quanto os trabalhos nos Estados Unidos. Desde o início dos anos 2000 vários segmentos da CE vêm realizando estudos que resultam em relatórios densos e significativos sobre o monopólio das editoras comerciais, o custo crescente das publicações e o fechamento do sistema.

Exemplares são as iniciativas do European Bureau of Library, Information and Documentation Associations (EBLIDA) ${ }^{12}$ e do European Research Consortium for Informatics and Mathematics (ERCIM), ${ }^{13}$ com a publicação, respectivamente, de Statement Towards an Effective Scientific Publishing System for European Research (2005) e Statement on Open Access (2006), em favor do acesso aberto. Ambos os documentos rejeitam o modelo tradicional (comercial) de publicação e clamam por mudanças e por políticas que tornem acessíveis os resultados de pesquisa por meio do depósito em repositórios digitais

Em março de 2006 o Study on the economic and thecnical evolution of the scientific publication markets in Europe, aponta para os prejuízos que resulta para o sistema de informação em ciência e tecnologia, o modelo dominante de publicação científica, baseado nos oligopólios das editoras comerciais. O relatório estabelece como uma das ações necessárias, a adoção, na Europa, de política de obrigatoriedade de acesso do público - o mais rápido possível após a publicação - aos resultados das pesquisas financiadas por verbas oriundas dos cofres públicos. Recomenda ainda que os países-membro e as instituições e associações científicas da Comunidade Europeia devem estudar a viabilidade dessa política e da implementação de repositórios digitais (EUROPEAN COMMISSION, 2006).

\footnotetext{
${ }^{11} \mathrm{http}: / /$ www.nsf.gov

12 http://www.sub.uni-goettingen.de/frankfurtgroup/openaccess/eblida.pdf

${ }^{13}$ http://www.ercim.org/publication/Ercim_News/enw64/ercim-oa.html
} 
O Digital Repository Infrastructure Vision for European Research (DRIVER), ${ }^{14}$ lançado em 2006, é resultado desse estudo citado. Inspirado no DAREnet ${ }^{15}$ da Holanda, esse grande projeto, esforço conjunto da Comissão Europeia e nove instituições de pesquisa, tem o objetivo de auxiliar os países da Comunidade Europeia a implementar seus repositórios digitais para promover o desenvolvimento de infraestrutura de conhecimento adequada para as pesquisas da Comunidade.

Há outro importante documento, tornado público em fevereiro de 2007, em que a Comissão Europeia declara seu compromisso com o acesso, a disseminação e a preservação de conteúdos digitais, enfatizando a necessidade de iniciativas que levem à disseminação ampla da informação científica. Propõe também algumas ações como: a) acesso a resultados de pesquisa financiados pela Comunidade; b) financiamento de projetos e infraestrutura de pesquisa - especialmente repositórios; c) alimentação de futuros debates; d) coordenação política e debate político com os interessados (COMMISSION OF THE EUROPEAN COMMUNITIES, 2007).

No âmbito da Comunidade Europeia, várias ações foram e vêm sendo desenvolvidas. Em consequência, há uma série de declarações em favor do acesso aberto às publicações científicas - como as emanadas do próprio Parlamento Europeu. As políticas oficialmente estabelecidas dentro da própria estrutura da Comunidade são também cada vez mais frequentes - por exemplo, pelo Scientific Council of the European Research Council ou o European Research Council - e no âmbito dos estados-membro do bloco.

\section{REINO UNIDO}

As discussões em torno do acesso aberto sempre estiveram presentes na agenda da comunidade científica do Reino Unido, de onde partiram várias iniciativas e resultados representativos das ações em prol da filosofia aberta. São exemplos disso o software EPrints, de 2000, desenvolvido na Universidade de Southampton, os projetos RoMEO (Rights MEtadata for Open archiving) e SHERPA (Securing a Hybrid Environment for Research Preservation and Access), ambos criados em 2002 pelo Joint Information Systems Committee (JISC). Trabalhando com programas de pesquisa e inovação sobre o uso de tecnologias de informação no ensino, aprendizagem e pesquisa, o Committee é uma organização fundamental para o desenvolvimento do acesso aberto no Reino Unido, porque propõe ações e dá suporte às iniciativas nacionais. ${ }^{16}$

Em razão do trabalho do JISC, outras atuações tornaram-se factíveis no Reino Unido. Além disso, ele provê os órgãos legislativos e executivos, de dados resultantes de investigações, subsidiando assim futuras leis e políticas oficiais regulatórias.

\footnotetext{
${ }^{14} \mathrm{http}$ ///www.driver-repository.eu/index.php?option=com_frontpage\&ltemid=1

${ }^{15} \mathrm{http}: / /$ www.narcis.info

${ }^{16}$ http://www.jisc.ac.uk/whatwedo.aspx
} 
Em dezembro de 2003 o Science and Technology Committee do Parlamento Britânico (House of Commons) divulga a futura realização de uma enquete sobre preços e acessibilidade de publicações científicas, cujo relatório final, denominado Scientific publications: free for all? apresenta 82 itens, entre evidências sobre a situação do sistema de publicações científicas no Reino Unido e recomendações em torno da necessidade de estabelecimento de modelos mais eficazes de acesso do contribuinte a essas informações (HOUSE OF COMMONS, 2003). Na sequência, um grupo de sete pesquisadores e professores das Universidades de Southampton e Loughborough e do eScience, assinaram e tornaram públicas as Recommendations for UK Open-Access Provision Policy: to UK Government Science and Technology Committee, ${ }^{17}$ em que argumentam, discutem aquele relatório e propõem linhas de ação em nível governamental.

Há, efetivamente, a partir desses fatos, uma série de declarações, recomendações e reconhecimento, pelos órgãos governamentais do Reino Unido, da necessidade de abertura de acesso aos resultados de pesquisa como:

- A Scottish Declaration of Open Access (2004);

- As posições indicadas pelo documento assinado por cinco dos oito Research Councils UK (RCUK) (2006);

- Em 2006 o Wellcome Trust solicita que os pesquisadores por ela financiados depositem seus trabalhos no PubMed Central (MATSUBAYASHI et al., 2006; WELLCOME TRUST, 2008);

- Em janeiro de 2007 o UK PubMed Central foi oficialmente indicado pelo grupo fundador - um misto de organizações governamentais e não-governamentais -, como o repositório de acesso aberto, e obrigatório, das pesquisas financiadas por elas. O repositório abriga quase dois milhões de artigos em textos completos, segundo dados de dezembro de 2009 (UK PUBMED CENTRAL, 2009).

\section{ALEMANHA}

Ocorreu na Alemanha, em 2003, uma das principais manifestações da iniciativa de acesso aberto. A Declaração de Berlin, elaborada e tornada pública em reunião promovida pela Sociedade Max Planck, teve como signatárias várias organizações internacionais e é um marco importante para o movimento em defesa da abertura da informação científica.

A iniciativa alemã mais importante ocorre na Deutsche Forschungsgemeinschaft (DFG) (Fundação Alemã de Pesquisa), órgão governamental que promove e financia pesquisas no âmbito das universidades e institutos de pesquisa do país. Signatária da Declaração de Berlin, em

\footnotetext{
${ }^{17}$ http://users.ecs.soton.ac.uk/harnad/Temp/UKSTC.htm
} 
2006 a DFG adotou uma política que recomenda aos pesquisadores que os resultados de pesquisas financiadas pela Fundação sejam publicados em meio digital com acesso aberto. $O$ texto da política não obriga, apenas recomenda que os pesquisadores depositem, em repositórios específicos, trabalhos já publicados em meios convencionais, ou publiquem em periódicos científicos de acesso aberto (DEUTSCHE FORSCHUNGSGEMEINSCHAFT, 2006). Em 2007, entretanto, ações mais específicas foram realizadas por um programa de financiamento voltado para a expansão e aprimoramento de periódicos científicos que adotassem a política de acesso aberto da DFG (SUBER, 2007).

Também em 2007 a associação civil Netzwerk Freies Wissen, aproveitando-se de reunião do G8 em Munique, tornou público o documento For better development and just access to knowledge in all forms, em que aponta as inquietações do grupo com questões relativas às restrições de acesso a: informações, produtos da biodiversidade, remédios, ciência, softwares. Demonstra também preocupação com as restrições de uso e cópia de conteúdos digitais (FOR BETTER..., 2009).

No âmbito do Parlamento Alemão (Bundesrat) há uma lei em tramitação e uma comunicação, de 2007, favorável à posição da Comissão Europeia sobre a disseminação da informação científica, além de apelo em prol do acesso aberto, rápido e o mais livre possível a essa informação (LE LIBRE..., 2009).

\section{FRANÇA}

Na França, as iniciativas em favor do acesso livre remontam ao final da década de 1990, a exemplo do portal Revues.org, ${ }^{18}$ que reúne revistas da área de Ciências Sociais e Humanas, coordenado pelo Centre pour l'Édition Électronique Ouverte (CLEO). ${ }^{19} \mathrm{Na}$ sequencia, em 2001, o Centre National de la Recherche Scientifique (CNRS) ${ }^{20}$ lança o repositório multidisciplinar Hyper Articles en Ligne $(\mathrm{HAL}){ }^{21}$

Por ser a maior e mais importante instituição de pesquisa do país, o CNRS, por intermédio de seus departamentos, laboratórios e institutos, tem papel essencial nos caminhos do acesso aberto na França. Várias iniciativas dispersas e isoladas foram descontinuadas até que o CNRS anunciou, no início de 2005, a política oficial do órgão e a criação do Directorate for Scientific Information (DIS), medidas que evidenciam o interesse do CNRS em relação às questões do acesso aberto e a posição estratégica da instituição para a comunidade francesa. Ainda nesse ano, o CNRS, o Institut National de la Santé e de la Recherche Medicale (INSERM), o Institut National de Recherche Agronomique (INRA) e o Institut National de Recherche en Informatique et en Automa-

\footnotetext{
${ }^{18}$ http://www.revues.org/

${ }^{19}$ http://cleo.cnrs.fr/

${ }^{20}$ http://www.cnrs.fr/

${ }^{21} \mathrm{http}: / /$ hal.archives-ouvertes.fr/
} 
tique (INRIA) anunciaram uma política conjunta por intermédio da implantação de repositórios de resultados de pesquisas. Esses repositórios de acesso aberto contemplariam os requisitos técnicos de interoperabilidade, de modo a proporcionar maior visibilidade aos trabalhos dos pesquisadores (GRUTTEMEIER, 2006; JOLY, 2007).

Em novembro de 2007 a Agence Nationale de la Recherche (ANR) publica uma nota requerendo que todas as todas as publicações - existentes e futuras - resultantes de pesquisas financiadas pela agência sejam depositadas no repositório HAL, respeitadas as regras de propriedade intelectual - literária, artística e intelectual - e as regras de confidencialidade relativas às pesquisas (AGENCE NACIONAL DE RECHERCHE, 2007).

\section{AUSTRÁLIA}

Na Austrália, a iniciativa que merece destaque é a Declaração de Acesso Aberto, de responsabilidade do Australian Research Information Infrastructure Committee (ARIIC), lançada em dezembro de 2004. O Committee tem o objetivo de promover o desenvolvimento de infraestrutura de informações coerentes para o ensino superior australiano, por meio de sistemas de publicação e comunicação acadêmica, visando a disseminação dos resultados de pesquisa, bem como a preservação dessas informações.

No que se refere ao acesso aberto, o ARIIC reconhece que as comunicações digitais propiciam oportunidades mais eficientes e efetivas para o desenvolvimento de sistemas de publicações acadêmicas. Além disso, salienta que o acesso aberto aos resultados de pesquisa realça o perfil das universidades e programas de pesquisas nacionais, contribuindo para o avanço do conhecimento; mantêm a qualidade e autoridade da publicação acadêmica, sendo que a integridade do registro erudito é de importância crítica para qualquer evolução e possibilita a construção de comunidades informadas, colaborando para a exclusão das desvantagens sociais e econômicas (AUSTRALIAN RESEARCH..., 2004).

De acordo com as intenções da Declaração, o ARIIC se propõe a desenvolver ações para: construir infraestrutura para o avanço do acesso aberto, como por exemplo, repositórios institucionais; estimular a conscientização em relação aos princípios e práticas do acesso aberto; implementar políticas públicas que assegurem os direitos autorais no uso da informação com fins educacionais e de pesquisa; e cooperar com o governo australiano no sentido de melhorar o acesso às informações, maximizando a informação de domínio público (AUSTRALIAN RESEARCH..., 2004).

Outras organizações australianas envolvidas com ações e iniciativas para o acesso aberto que podemos destacar são: a Australian National University, a Australian Government Productivity Commission, o Australian Research Council, o Australia's Department of Education, Science and 
Training, o Australian Group of Eight e o Australian Partnership for Sustainable Repositories (SUBER, 2007).

\section{CANADÁ}

No que se refere às políticas de acesso aberto no Canadá, salientamos a iniciativa do Canadian Institutes of Health Research $(\mathrm{CIRH})$, que em setembro de 2007, lança a política de acesso aos resultados das pesquisas. O objetivo dessa política é melhorar o acesso às produções de pesquisas subsidiadas pelo $\mathrm{CIRH}$ e aumentar a difusão de seus resultados. De acordo com o $\mathrm{CIRH}$, o acesso aberto possibilita o alcance de um público muito mais amplo, aumentando potencialmente o impacto das pesquisas, bem como a aplicação de seus resultados (CANADIAN INSTITUTES..., 2007).

Várias outras organizações canadenses propuseram iniciativas de apoio ao acesso aberto à informação, dentre elas, destacamos: National Library of Canadian, Canadian Library Association, Library and Archives Canada, Canada's Athabasca University, AlouetteCanada, Canadian Breast Cancer Research Alliance, Canada's Social Science and Humanities Research Council (SUBER, 2007).

\section{PORTUGAL}

Em Portugal a Declaração de Estoril sobre o Acesso à Informação, ${ }^{22}$ lançada durante o $8^{\circ}$ Congresso Nacional de Bibliotecários, Arquivistas e Documentalistas, em maio de 2004, no Estoril, reconhece e apoia as definições, objetivos e princípios do acesso aberto, tal como definidos na Declaração de Budapest Open Access Initiative e na Declaração de Berlim sobre o Acesso ao Conhecimento nas Ciências e Humanidades. Vale também ressaltar que na declaração os profissionais fazem uma recomendação ao governo português quanto à obrigatoriedade da disponibilização, por meio do acesso livre, dos resultados das pesquisas financiadas com o dinheiro público. Além disso, solicitam às universidades, laboratórios, centros de pesquisa, sociedades científicas e demais organizações que produzem, financiam ou editam literatura científica em Portugal, que elaborem políticas visando a promoção do acesso aberto (DECLARAÇÃO..., 2004).

Outra iniciativa de Portugal é o Compromisso do Minho: Compromisso sobre o Acesso Livre à Informação Científica em Países Lusófonos, ${ }^{23}$ proposto na $2^{\text {a }}$ Conferência sobre Acesso Livre ao Conhecimento, realizada em novembro de 2006, em Portugal. Os proponentes desse documento comprometem-se a solicitar às instituições acadêmicas e científicas o envolvimento no que se refere às iniciativas no âmbito do acesso aberto que visam: contribuir para aumentar o im-

\footnotetext{
${ }^{22}$ http://www.apbad.pt/Downloads/DeclaracaoEstoril.pdf

${ }^{23}$ http://www.ibict.br/alemplus/arquivos/compromisso.pdf
} 
pacto global da produção científica originada nos países lusófonos por meio do acesso aberto, canalizar os esforços de pesquisa e desenvolvimento nos países lusófonos para uma ação integrada no que tange às iniciativas de acesso aberto, bem como, sensibilizar as instituições governamentais e as agências de financiamento para iniciativas que promovam o acesso aberto (COMPROMISSO..., 2006).

De mais a mais, visando operacionalizar as propostas contidas no Compromisso do Minho, seus idealizadores decidiram formar um Grupo de Trabalho, com três grandes linhas de ação: a) sensibilização política; b) cooperação em pesquisa e desenvolvimento; e c) disseminação de informação sobre o acesso livre na comunidade lusófona.

\section{OUTRAS INICIATIVAS: CHINA, RÚSSIA, ÍNDIA, BÉLGICA, ÁFRICA DO SUL}

Além das diversas iniciativas dos países mencionados no decorrer deste trabalho, podemos ainda citar a China, a Rússia, a Índia, a Bélgica e a África do Sul que também vêm tornando públicas algumas propostas e ações.

A China, em outubro de 2006, anunciou alguns planos para promover o acesso aberto à informação. De acordo com o ministro de Ciência e Tecnologia desse país à época, mais de $80 \%$ dos dados referentes à pesquisa da China em ciência pura - como matemática teórica, física e química - estariam livremente disponíveis na Internet naquele ano. O Ministro afirmava ainda que até 2010, a China pretende estabelecer 40 centros de dados científicos, cobrindo 300 bases de dados referentes a meio ambiente, agricultura, saúde humana, ciência pura, engenharia e informações de tecnologia. Todas essas bases estarão disponíveis por meio de um portal de acesso livre promovido pelo Ministério de Ciência e Tecnologia (JIA, 2006).

Na Rússia, o Central Economics and Mathematics Institute of Russian Academy of Sciences decretou que todos os seus pesquisadores deverão depositar os resultados de suas pesquisas em repositório institucional de livre acesso (CEMI..., 2008).

De acordo com dados do Registry of Open Access Repository Material Archiving Policies (ROARMAP),${ }^{24}$ na Índia, a Bharathidasan University, a partir de novembro de 2006, tornou obrigatório o envio da produção científica de seus pesquisadores para o repositório institucional da universidade. A Bharathidasan University acredita que o repositório aumentará a citação de suas publicações e impulsionará a colaboração na pesquisa interdisciplinar. O ROARMAP registra também as ações da Madurai Kamaraj University, do National Institute of Technology e do Council of Scientific \& Industrial Research.

Além dessas iniciativas Suber (2007) lista, no Timeline of the Open Access Movement, outras organizações que manifestaram apoio ao acesso aberto à informação científica na Índia, co-

\footnotetext{
${ }^{24}$ http://www.eprints.org/openaccess/policysignup
} 
mo por exemplo, a Working Group on Libraries for India's National Knowledge Commission e a India's National Centre for Science Information.

Em relação à Bélgica, os dados do ROARMAP, indicam que a Research Foundation Flanders, segue a Declaração de Berlim (2003) para a promoção de acesso aberto à informação científica e exige que os pesquisadores que recebem subsídios para os seus estudos devem depositar sua produção científica em um banco de dados de acesso aberto ao público, visando um maior impacto e valorização de seus trabalhos. Outra é o Open Marine Archive (OMA), coodernado pelo Flanders Marine Institute, que disponibiliza o acesso aberto à coleção digital dos trabalhos dos investigadores marinhos, com o objetivo de aumentar a visibilidade, distribuição e uso dos resultados das pesquisas, bem como, melhorar a comunicação científica.

$\mathrm{Na}$ África do Sul podemos destacar duas iniciativas, ocorridas em 2006. A primeira diz respeito a um relatório elaborado pela Academy of Science of South Africa, em março de 2006, que recomenda o uso dos dois modelos de acesso aberto à informação - golden road (via dourada) e green road (via verde). A segunda iniciativa ocorreu em abril de 2006, quando o Committee on Data for Science and Technology (CODATA) publicou o relatório do workshop internacional, realizado em setembro de 2005, em Pretória, sobre as estratégias para o acesso permanente às informações científicas na África do Sul, com enfoque na saúde e informações ambientais para o desenvolvimento sustentável. Esse relatório alerta para a urgência de as instituições africanas aderirem ao acesso aberto, promovendo o compartilhamento de dados (ARNOLD; ANDERSON; UHLIR, 2006; SUBER, 2007).

\section{CONSIDERAÇÕES FINAIS}

Os resultados desta pesquisa indicam que o movimento de acesso livre à informação científica é preocupação oficialmente registrada em vários países, embora com diferentes graus de desenvolvimento e diferentes formas de ação.

Uma das diferenças diz respeito às próprias determinações das políticas, pois algumas obrigam pesquisadores e instituições públicas de pesquisa a disponibilizarem, em acesso aberto, aos resultados de sua produção, enquanto outras apenas sugerem o envolvimento e a participação desses pesquisadores e instituições no movimento. Termos e expressões com significados próximos fazem diferença considerável na prática das políticas. Por exemplo, um texto que apenas solicite (request, solliciter) o depósito - considerado, neste caso, depósito voluntário - estará sempre sujeito à vontade do pesquisador; outro que obrigue (mandate, demander), embora levante polêmica e possa ser considerado autoritário, resultará em ações mais efetivas.

Este estudo evidencia ainda a presença de organizações não-governamentais e organismos de governos - que não atuam diretamente no âmbito legislativo - na liderança do movimento, impulsionando, inclusive a participação de órgãos governamentais na oficialização de políticas e 
regulamentação de leis, como é o caso do IBICT no Brasil. É por iniciativa do IBICT que está em tramitação - por longo tempo, é verdade - um projeto de Lei (PL 1120/2007) que dispõe sobre o processo de disseminação da produção técnico-científica pelas instituições de ensino superior e pesquisa no Brasil, demonstrando a liderança e capacidade desse Instituto em elaborar e coordenar programas e ações de incentivo para que as instituições de ensino e pesquisa de todo o país disponibilizem, por meio do acesso aberto, sua produção científica.

É importante destacar que, em muitos países, as primeiras iniciativas oficiais partiram de instituições de pesquisa na área médica. É provável que isto se deva ao fato de ser esta uma das áreas pioneiras na organização e compartilhamento de recursos de informação em meio eletrônico. Além disso, os resultados das pesquisas da área médica têm, em sua maioria, grande visibilidade em razão da função social.

\section{REFERÊNCIAS}

AGENCE NACIONAL DE RECHERCHE. L'ANR incite les chercheurs à intégrer leurs publications dans le système d'archives ouvertes. 2007. Disponível em: <http://www.agence-nationalerecherche.fr/actualite/13? Inglnfold=159> Acesso em: 14 maio 2008.

ALLIANCE FOR TAXPAYER ACCESS. 2009. Disponível em:

<http://www.taxpayeraccess.org/index.html> Acesso em: 23 dez. 2009.

AN OPEN letter to the U.S. Congress signed by 25 Nobel Prize winners. 2005. Disponível em: <http://www.fas.org/sgp/news/2004/08/nobel082604.pdf> Acesso em: 10 abr. 2008.

ARNOLD. R.; ANDERSON, W.; UHLIR, P. Strategies for permanent access to scientific information in Southern Africa: focus on health and environmental information for sustainable development. Pretoria: CODATA, 2006. Disponível em: <http://stardata.nrf.ac.za/html/CodataReport.pdf>. Acesso em: 29 maio 2008.

ASSOCIATION OF RESEARCH LIBRARY. Public policies: public access policies. Disponível em: <http://www.arl.org/pp/access/index.shtml> Acesso em: 27 dez. 2009.

AUSTRALIAN RESEARCH INFORMATION INFRASTRUCTURE COMMITTEE. Open access statement. 2004. Disponível em:

<http://www.caul.edu.au/scholcomm/OpenAccessARIICstatement.doc>. Acesso em: 28 maio 2008.

CANADIAN INSTITUTES OF HEALTH RESEARCH. Policy on access to research outputs. 2007. Disponível em: <http://www.cihr-irsc.gc.ca/ e/34846.html>. Acesso em: 28 maio 2008.

CEMI Ras joins an international initiative open access to research. Disponível em: <http://www.cemi.rssi.ru/rus/news/initiat-eng.htm>. Acesso em: 29 maio 2008.

COMMISSION OF THE EUROPEAN COMMUNITIES. Communication from the Commission to the European Parliament, the Council and the European Economic and Social Committee on scientific information in the digital age: access, dissemination and preservation. 2007. Disponível em: $<\mathrm{http}: / /$ ec.europa.eu/research/science-society/document_library/pdf_06/communication022007_en.pdf> Acesso em: 22 dez. 2009. 
COMPROMISSO do Minho compromisso sobre o acesso livre à informação científica em países lusófonos. 2006. Disponível em: <http://www.ibict.br/alemplus/arquivos/compromisso.pdf>. Acesso em: 29 maio 2008.

COSTA, S. Filosofia aberta, modelos de negócios e agências de fomento: elementos essenciais a uma discussão sobre o acesso aberto à informação científica. Ciência da Informação, v. 35, n. 2, p. 39-50, maio/ago., 2006. Disponível em: <http://www.ibict.br/cionline/viewarticle.php?id=926> Acesso em: 23 fev. 2007.

DECLARAÇÃO do Estoril sobre o acesso à informação. 2004. Disponível em: <http://www.apbad.pt/Downloads/DeclaracaoEstoril.pdf>. Acesso em: 28 maio 2008.

DEUTSCHE FORSCHUNGSGEMEINSCHAFT. DFG passes open access guidelines. 2006. Disponível em:

<http://www.dfg.de/en/news/information_science_research/other_news/info_wissenschaft_04_06.h tml> Acesso em: 12 fev. 2008.

EUROPEAN COMMISSION. Directorate-General for Research Information and Communication Unit. Study on the economic and thecnical evolution of the scientific publication markets in Europe, 2006. Disponível em: <http://ec.europa.eu/research/science-society/pdf/scientific-publicationstudy_en.pdf> Acesso em: 17 dez. 2009.

FOR better development and just access to knowledge in all forms. Against the domination of exclusionary rights on the knowledge economy. A civil society statement concerning intellectual property and the 2007 G8 summit. 2007. Disponível em: < http://www.siug.ch/URG/declaration2007-05-23.pdf> Acesso em: 12 dez. 2009.

GRUTTEMEIER, H. The way to Open Access: French strategies to move forward. Library and Information Service, v. 50, n. 1, p. 27-33, 2006. Disponível em:

<http://eprints.rclis.org/archive/00005888/01/HG_Article_Chine_3.pdf> Acesso em: 14 dez. 2009.

HOUSE OF COMMONS. Science and Technology Committee. Scientific Publications: Free for All? Tenth Report of Session 2003-04. London: House of Commons, 2004. Disponível em: <http://www.publications.parliament.uk/pa/cm200304/cmselect/cmsctech/399/399.pdf> Acesso em: 20 maio 2008.

IFLA. Statement on open access to scholarly literature and research documentation. 2003. Disponível em: < http://www.ifla.org/V/cdoc/open-access04.html>. Acesso em: 29 maio 2008.

JIA, H. China unveils plans to boost scientific data sharing. 2006. Disponível em: <http://www.scidev.net/en/news/china-unveils-plans-to-boost-scientific-data-shari.html>. Acesso em: 29 nov. 2009.

JOLY, M. Les archives ouvertes en France. Journées ABES, 2007. Disponível em: <http://www.abes.fr/abes/documents/reseau/journees_reseau/session1_AO.pdf> Acesso em: 14 maio 2008.

JOSIAH, J. Foreword. In: DAS, Anup Kumar. Open access to knowledge and information: scholarly literature and digital library initiatives; the South Asian scenario. New Delhi: Unesco, 2008. Disponível em: <http://unesdoc.unesco.org/images/0015/001585/158585e.pdf> Acesso em: 11 maio 2008.

LE LIBRE accès à l'information scientifique et technique. Brèves. 2008. Disponível em: <http://openaccess.inist.fr/archivesbreves.php3> Acesso em: 12 dez. 2009. 
MATSUBAYASHI, M. et al. The current status of Open Access in Biomedical field: the comparison of countries relating to the impact of national policies. In: ASIS\&T ANNUAL MEETING, 69., Austin. Proceedings...Austin, 2006.

NATIONAL SCIENCE FOUNDATION. Cyberestructure Council. NSF's cyberinfrastructure vision for 21st Century discovery. 2006. Disponível em: <http://www.nsf.gov/od/oci/ci_v5.pdf> Acesso em: 12 dez. 2009.

RESEARCH COUNCILS UK. Research Councils UK' updated position statement on access to research outputs. 2006. Disponível em:

<http://www.rcuk.ac.uk/cmsweb/downloads/rcuk/documents/2006statement.pdf> Acesso em 27 maio 2008.

SCOTTISH Declaration of Open Access. 2004. Disponível em: <http://scurl.ac.uk/WG/OATS/declaration.htm> Acesso em: 27 maio 2008.

SUBER, P. Open Access in the United States. In: JACOBS, N. (Ed.). Open access: key strategic, technical and economic aspects. [s.I.]: Chandos Publishing, 2006. Disponível em: <e-

prints.rclis.org/archive/00006671/01/Suber_2006.pdf> Acesso em: 10 dezembro 2009.

SUBER, P. Timeline of the Open Access Movement. 2007. Disponivel em:

<http://oad.simmons.edu/oadwiki/Timeline_2007>. Acesso em: 12 dez. 2009.

UK PubMed Central: free archive of life sciences journals. Disponível em: <http://ukpmc.ac.uk/> Acesso em: 12 dez. 2009.

WELLCOME TRUST. Position statement in support of open and unrestricted access to published research. 2008. Disponível em: <http://www.wellcome.ac.uk/About-us/Policy/Spotlightissues/Open-access/Policy/index.htm> Acesso em: 28 maio 2008.

\section{Title}

Open Access to Scientific Information: governmental policies and initiatives

\section{Abstract}

This article discusses policies concerning the open access to scientific information and also presents some strategies emphasizing the governmental initiatives in different countries. It describes Unesco's and IFLA's recommendations and identifies the main international initiatives, actions and recommendations. Finally are demonstrated some peculiarities related to the areas and to the different possibilities to handle with the issues covered by the policies that are already established.

\section{Keywords}

Open Access. Scientific Information. Information Policies.

\section{Titulo}

Acceso abierto a la información científica: políticas e iniciativas gobernamentales 


\section{Resumen}

El artículo discute políticas referentes al acceso abierto a la información científica y también presenta algunas estrategias que acentúan las iniciativas gobernamentales en diversos países. Describe las recomendaciones de la Unesco y de la IFLA. Se identifican las principales iniciativas, las acciones y las recomendaciones internacionales. Finalmente se demuestran algunas particularidades relacionadas con las áreas y con las diversas posibilidades para dirigir con las ediciones cubiertas por las políticas que ya se establecieron.

\section{Palabras Clave}

Acceso abierto. Información científica. Políticas de información.

Recebido em: 26.12.2009

Aceito em: 10.02.2010 